\title{
Letter to Editor: The Low Prevalence of Hepatitis C Virus and Human Immunodeficiency Virus Coinfection and Hepatitis C Virus Mono-Infection Among Methadone Toxicity Patients
}

Haleh Talaie $^{1^{*}}$ (D), Atieh Mousavizadeh ${ }^{1}$ (D), Behjat Barari ${ }^{1}$ (D)

1 Toxicological Research Center, Department of Clinical Toxicology, Loghman-Hakim Hospital, Shahid Beheshti University of Medical Sciences, Tehran, Iran.

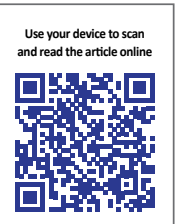

Citation: Talaie H, Mousavizadeh A, Barari B. The Low Prevalence of Hepatitis C Virus and Human Immunodeficiency Virus Coinfection and Hepatitis C Virus Mono-Infection Among Methadone Toxicity Patients. International Journal of Medical Toxicology and Forensic Medicine. 2020; 10(1):28457. https://doi.org/10.32598/ijmtfm.v10i1.28457

dol https://doi.org/10.32598/ijmtfm.v10i1.28457

\section{To the Editor}

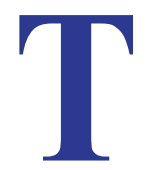

he incidence of Hepatitis C Virus (HCV) and Human Immunodeficiency Virus (HIV) coinfection in People Who Inject Drugs (PWIDs) is a public health issue; it presents various contentions to the healthcare providers. Although antiretroviral therapy improved the life expectancy of HIV-infected people, HCV-related mortality turned into a greater concern among these individuals [1]. According to Yen et al. 0.4\% of PWIDs were positive for $\mathrm{HIV}, 78 \%$ for $\mathrm{HCV}$, and $13.1 \%$ for $\mathrm{HIV} /$ HCV coinfections. Furthermore, they mentioned, "injecting drug users in the Taipei methadone maintenance treatment program had a very high prevalence of HIV/ HBV coinfection and HCV mono-infection"[2].

Besides, a systematic review and meta-analysis results suggested a high frequency of HIV/HBV coinfection $(>80 \%)$ in Intravenous (IV) drug users [3]. Although the incidence of HIV among IV drug users has been decreased, HCV is still endemic in this population [4]. We aimed to estimate $\mathrm{HCV} / \mathrm{HIV}$ coinfection frequency and its related risk factors among methadone poisoned patients who were admitted to the Loghman Hakim poisoning center between March 2012 and March 2017. Loghman-Hakim Hospital is a unique poisoning referral center in Tehran, Iran, that admits patients from all cities in Tehran Province, Iran. Annually, around 20000 hospitalized patients are observed and treated in this center, with 80-100 patients daily turn-over.

The required data were collected using a questionnaire, clinical examinations, and laboratory findings. The patients with a history of infectious diseases, like hepatitis $\mathrm{B}$ or $\mathrm{C}, \mathrm{HIV}$, and IV drug consumption, were excluded from the present research. The obtained blood samples were screened for antibodies to HCV and HIV using a commercially available Enzyme-Linked Immunosorbent Assay (ELISA). Furthermore, the relevant urine samples were analyzed for the presence of methadone with a rapid test. Among 200 participants, 134 (67\%) were male, and $66(33 \%)$ were female with the age range of 1 to 83 years. The methadone serum levels of $129(64.5 \%)$ patients were positive, $39(19.5 \%)$ were negative, and $32(16 \%)$ patients were not examined due to the short duration of hospitalization (i.e. $<2$ days). Underlying diseases, such as noncommunicable diseases $(11 \%)$, psychotic disorders (1.5\%), and respiratory disease $(3.5 \%)$ were detected in 30 cases. Reactive HCV-antibodies, active HIV-antibodies, and HIV/ $\mathrm{HCV}$ coinfection were observed in $10(5 \%), 2(1 \%)$, and 2 ( $1 \%$ ) of the study subjects, respectively. Figure 1 shows the prevalence of $\mathrm{HCV}$ and $\mathrm{HIV}$ infection by gender and age.

* Corresponding Author:

Haleh Talaie, MD, MPH

Address: Toxicological Research Center, Department of Clinical Toxicology, Loghman-Hakim Hospital, Shahid Beheshti University of Medical Sciences, Tehran, Iran.

Tel: +98 (912) 2394067

E-mail:talaie@sbmu.ac.ir 


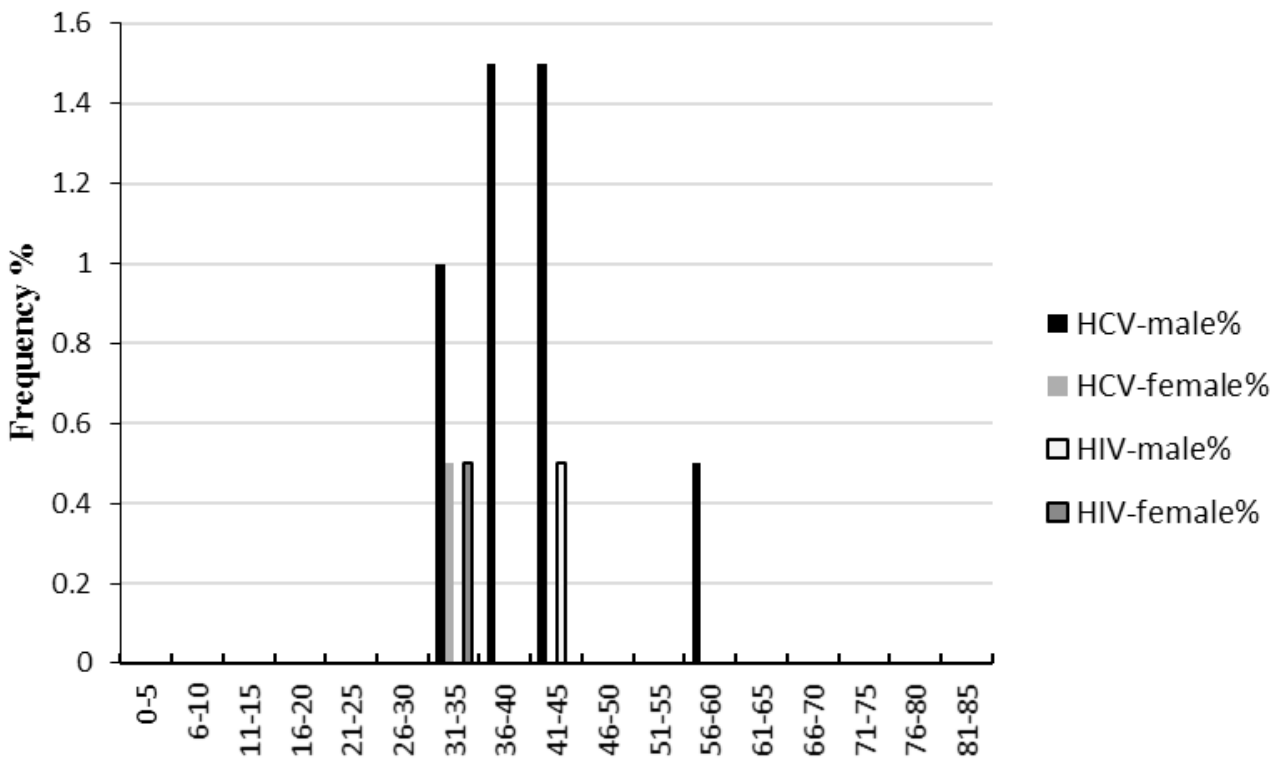

Age group, years

International Journal of

Figure 1. The prevalence of HCV and HIV infection by gender and age in Loghman Hakim poisoning center between March 2012 and March 2017

One of the HIV/HCV co-infected individuals was a homeless 40-year-old man with a hospitalization duration of 4 days; the other was a 32-year-old woman (47 hospitalization days) whose their hospital stay duration did not significantly differ from that of others, in this regard. Despite the study of Yen et al. for estimating HIV, $\mathrm{HCV}$, and $\mathrm{HIV} / \mathrm{HCV}$ coinfection, the rate of these infections among methadone poisoned cases in Loghman Hakim poisoning center, as a unique referral center, was surprisingly not significant. Thus, further research is required in this respect.

\section{Ethical Considerations}

\section{Compliance with ethical guidelines}

The study protocol was approved by the Ethics Review Committee in Research Deputy Department of Shahid Beheshti University of Medical Sciences, Tehran, Iran. [Ethical Code; IR.SBMU.RETECH.REC.1396.1356]

\section{Funding}

This research did not receive any specific grant from funding agencies in the public, commercial, or not-forprofit sectors.

\section{Author's contributions}

Conceptualization, Date curation, Review and editing: Haleh Talaie; Writing original draft: Atieh Mousavizadeh; Data collection: Behjat Barari.

\section{Conflict of interest}

The authors declared no conflict of interest.

\section{Acknowledgements}

This study was supported by Toxicological Research Center, Loghman-Hakim Hospital, Shahid Beheshti University of Medical Sciences, Tehran, Iran.

\section{References}

[1] Anderson KB, Guest JL, Rimland D. Hepatitis C virus coinfection increases mortality in HIV-infected patients in the highly active antiretroviral therapy era: Data from the HIV Atlanta VA Cohort Study. Clinical Infectious Diseases. 2004; 39:1507-13. [DOI:10.1086/425360] [PMID]

[2] Yen YF, Yen MY, S u LW, Li LH, Chuang P, Jiang XR, et al. Prevalences and associated risk factors of HCV/HIV coinfection and $\mathrm{HCV}$ mono-infection among injecting drug users in a methadone maintenance treatment program in Taipei, Taiwan. BMC Public Health. 2012; 12:1066-72. [DOI:10.1186/1471-2458-12-1066] [PMID] [PMCID] 
[3] Platt L, Easterbrook P, Gower E, McDonald B, Sabin K, McGowan C, et al. Prevalence and burden of HCV coinfection in people living with HIV: A global systematic review and meta-analysis. The Lancet Infectious Diseases 2016; 16(7):797808. [DOI:10.1016/S1473-3099(15)00485-5]

[4] Talaie H, Shadnia S, Okazi A, Pajouhmand A, Hasanian $\mathrm{H}$, Arianpoor $\mathrm{H}$. The prevalence of hepatitis $\mathrm{B}$, hepatitis C and HIV infections in non-IV drug opioid poisoned patients in Tehran-Iran. Pakistan Journal of Biological Sciences. 2007;10(2):220-4. [DOI:10.3923/pjbs.2007.220.224] [PMID] 